\title{
Potential role of S-adenosylmethionine in osteosarcoma development
}

This article was published in the following Dove Press journal:

OncoTargets and Therapy

20 June 2016

Number of times this article has been viewed

\author{
Hui Shi ${ }^{1,2, *}$ \\ Wei-dong Mul,* \\ Bing Zhang ${ }^{3}$ \\ Tao Meng ${ }^{2}$ \\ Shou-tao Zhang ${ }^{2}$ \\ Dong-sheng Zhou' \\ 'Department of Traumatic \\ Orthopaedics, Shandong Provincial \\ Hospital Affiliated to Shandong \\ University, Jinan, ${ }^{2}$ Department of \\ Bone and Joint Surgery, ${ }^{3}$ Department \\ of Urology Surgery, Binzhou Medical \\ University Hospital, Binzhou, \\ Shandong, People's Republic of China \\ *These authors contributed equally \\ to this work
}

\begin{abstract}
The metastatic form of osteosarcoma is a life threatening one since it metastasizes to the lungs. The major cause of metastatic osteosarcoma is hypomethylation of numerous genes that undergo overexpression to enable the progression of the disease. In the present study, $S$-adenosylmethionine (SAM), a predominant methyl donor, was administered to find out its effects on osteosarcoma progression. As evidence of tumor suppression, the SAM-treated mouse tissue was analyzed histologically, which exemplifies the control that SAM has over abnormal cell proliferation, especially on primary osteosarcoma, but it lacks positive effects on metastatic osteosarcoma. At the molecular level, the successful inhibition of primary osteosarcoma was found to be associated with a lower expression of Sox2, a protein highly expressed in osteosarcoma stem cells, along with an upregulated expression of TCTP. The data suggest that the administration of SAM has a positive role in treating primary osteosarcoma, but it has no role in suppressing metastatic osteosarcoma. The decreased expression of Sox 2 together with upregulation of TCTP following SAM administration indicates that SAM has a control over primary osteosarcoma.

Keywords: TCTP, $S$-adenosylmethionine, K7M2 cells, Sox2, BALB/c mouse
\end{abstract}

\section{Introduction}

Osteosarcoma is the most common aggressive form of bone tumor that occurs in children, and adults $>50$ years. ${ }^{1}$ It occurs due to abnormal osteoid production and differentiation along with genomic instability. The progression of osteosarcoma is associated with numerous genes with diverse genomic alterations. ${ }^{2}$ Previously, surgical resection therapy was practiced in osteosarcoma patients, which resulted in poor prognosis. However, prominent chemotherapy was developed during the $1980 \mathrm{~s}$, which increased the 5-year survival rate of osteosarcoma patients to $50 \%-80 \%$. ${ }^{3,4}$ Since then, there has been no significant progress in therapeutic modalities as well as in prognosis in patients suffering with osteosarcoma. ${ }^{5}$ Especially, the patients with the metastatic form of osteosarcoma have only a 10\%-30\% longer survival rate, ${ }^{6}$ and others exhibit less response. In order to improve the survival rate of patients, a new therapeutic target should be put forward to investigate the antimetastatic activity, or an effective drug must be required to impede the progression of osteosarcoma to metastatic stage.

Besides many reasons like deletion, translocation, amplification, and other mutations that contribute to tumor development, ${ }^{7}$ epigenetic changes have also paved the way for the development of many cancers, including osteosarcoma. ${ }^{8-10}$ The major epigenetic changes consist of DNA methylation, histone modification, and chromosome remodeling. ${ }^{11} S$-adenosylmethionine (SAM), an important molecule in biochemistry that donates its methyl group for many biochemical reactions, ${ }^{12}$ also acts as a precursor for forming higher polyamines. The polyamine helps to condense and 
stabilize DNA that controls the normal function of cells. It has been reported that SAM has anticancer properties and it is used as a therapeutic agent to treat cancer. ${ }^{13,14}$ The role of methylation especially in the promoter region was thought to repress gene expression based on the specific cell type ${ }^{15}$ and in some cases methylation-mediated gene expression, but still the more precise role of methylation in gene expression remains unclear. Similarly, there is no report that associates SAM with osteosarcoma stem cells.

TCTP is an evolutionally conserved protein from yeast to human, ${ }^{16-19}$ and has a vital role in cell cycle, ${ }^{20,21}$ apoptosis, ${ }^{22}$ cytoskeleton remodeling, ${ }^{21}$ protein synthesis, ${ }^{23}$ immune response, ${ }^{17}$ development of normal body growth and development of the organisms, ${ }^{24}$ and cancer. ${ }^{22}$ In addition, it was reported that TCTP expression was elevated in cancer tissues and also during the regeneration of liver; ${ }^{25}$ besides the protein has a major role in tumor reversion. ${ }^{22-26}$ Hence, TCTP is considered as a target for cancer therapy.

In this study, we investigated the effects of exogenous SAM on cancer stem cells of the osteosarcoma mouse model. We analyzed the progression after SAM administration and we studied SAM administration counteraction using Sox2 and TCTP that are linked to osteosarcoma stem cells.

\section{Materials and methods}

\section{Experimental animals with osteosarcoma}

The animal use and care, along with the protocol carried out in this study, was approved by the institutional animal ethical committee at Binzhou Medical University Hospital. For developing an osteosarcoma mouse model, aggressive osteosarcoma-forming K7M2 cells were used. ${ }^{27}$ Five-monthold male BALB/c mice were injected with K7M2 cells $\left(10^{6}\right.$ cells $/ 20 \mu \mathrm{L}$ ) to establish primary and metastatic forms of tumor. Following injection, one set of mice was sacrificed on the third week to obtain primary tumor and another set was allowed up to 5 weeks to obtain the metastatic form of osteosarcoma. The mice were subjected to digital radiography to confirm tumor progression before sacrifice. For determining the effects of SAM on tumor development, the mice were administered SAM (100 mg/kg/day) by oral gavage from the 15 th day of injection of K7M2 cells.

\section{Immunohistochemistry}

For immunohistochemistry, the dissected tissues were initially fixed in $10 \%$ formalin solution and processed to get paraffinembedded tissue. The microtome was set at $6 \mu \mathrm{m}$, and the sections obtained were fixed on a microscopic glass slide. Following deparaffinization with xylene, they were hydrated with water. To block the endogenous peroxidase activity, the sections were immersed in freshly prepared $10 \% \mathrm{H}_{2} \mathrm{O}_{2}$ and $10 \%$ methanol in $1 \mathrm{X}$ phosphate-buffered saline (PBS) for 20 minutes. After washing with $1 \mathrm{XPBS}$, the sections were treated with $0.1 \%$ trypsin in $0.1 \% \mathrm{CaCl}_{2}$ at $37^{\circ} \mathrm{C}$ for 5 minutes. In order to facilitate binding of specific antibodies, the sections were incubated with the primary antibody overnight at $4{ }^{\circ} \mathrm{C}$ followed by the addition of a suitable secondary antibody for 30 minutes at room temperature. After washing the tissue sections, the slides were developed using a DAB (diaminobenzidine) kit as a chromogen for detecting signals. For immunohistochemistry experiments, the following primary antibodies were used: antiSox 2 antibody (ab97959) and anti-TCTP antibody (ab37506) from Abcam (Cambridge, UK). Similarly, the secondary antibody Goat Anti-Rabbit IgG H\&L (HRP) (ab6721) from Abcam was used to detect the signals.

\section{Western blot analysis}

Cell lysates were prepared from normal tissue as well as from the primary and metastatic forms of osteosarcoma tissue as per the protocol mentioned in the literature. ${ }^{28}$ The proteins were isolated and resolved in 10\% SDS-PAGE gel. They were then transferred to the membrane and incubated with the primary antibody (anti-Sox 2 antibody [ab97959] or anti-TCTP antibody [ab37506]) at a dilution ratio of 1:500. The unbound primary antibody was washed out and further incubated with the secondary antibody (Goat Anti-Rabbit IgG H\&L [HRP] [ab6721]) at a dilution ratio of 1:5,000. Later, the signals were visualized with the DAB kit.

\section{Results \\ Positive effects of SAM on the osteosarcoma mouse model}

In the present study, the mouse model of osteosarcoma was successfully initiated by injecting highly tumorigenic and metastatic osteosarcoma-forming K7M2 cells. The experimental mice responded well to injected K7M2 cells, which were examined through histological observation by comparing with the control mice (Figure 1A-C). Following injection, the mice were recognized with primary tumors, which were histologically visualized on the third week after injection of K7M2 cells (Figure 1B). Similarly, the metastatic condition of osteosarcoma was achieved on the fifth week after injection of K7M2 cells (Figure 1C). When compared with the control, which had an even arrangement of cells (Figure 1A), the primary and metastatic osteosarcoma cells were large and deeply stained together with a disorderly arrangement of cells (Figure 1B and $\mathrm{C}$ ). The unique feature of the metastatic form (Figure 1C) 

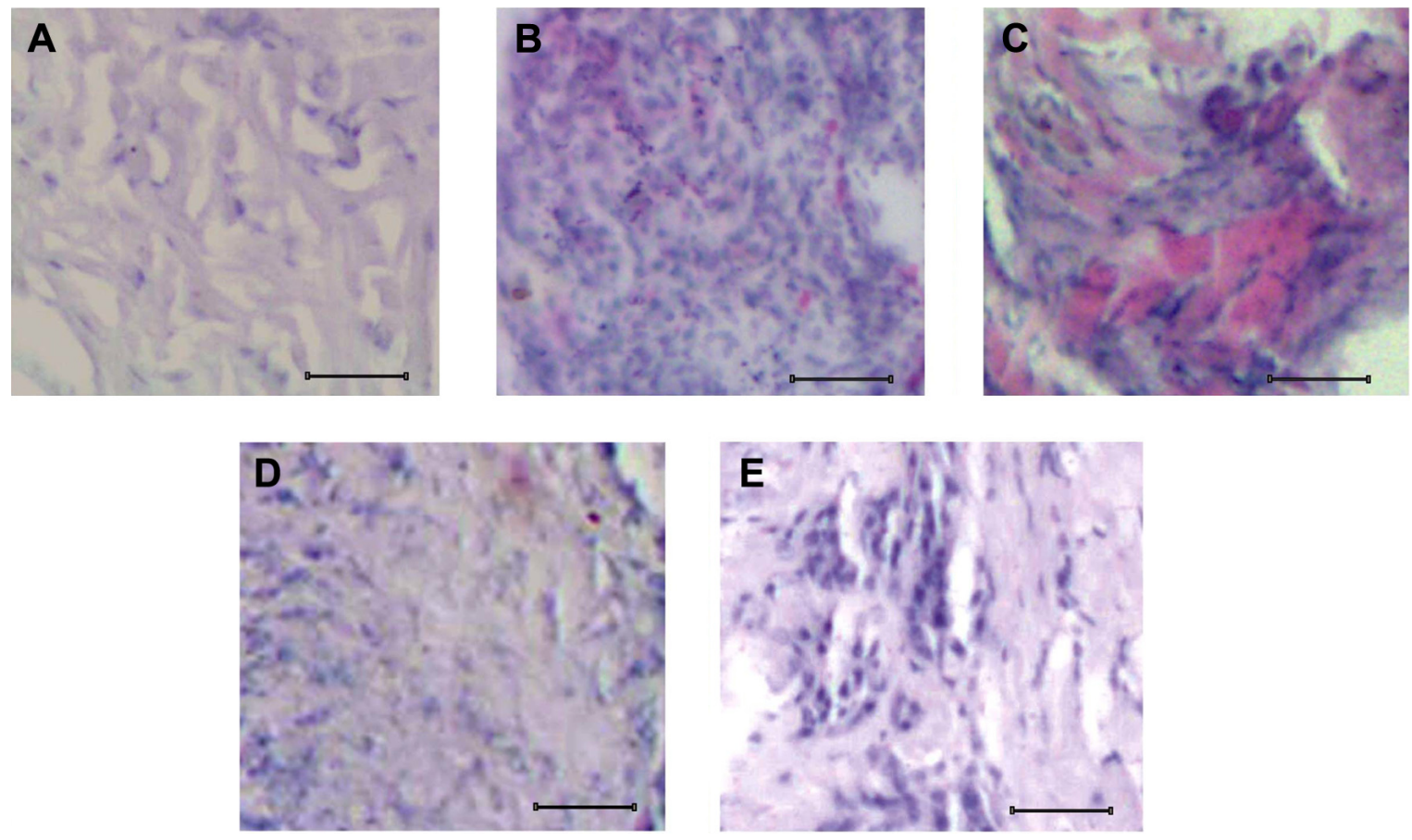

Figure I Histological characteristics of normal and osteosarcoma tissues.

Notes: (A) Histological section of normal bone tissue of a mouse showing uniform arrangement of cells. (B) Primary osteosarcoma tissue with abnormal mass of cells. (C) Metastatic osteosarcoma tissue showing irregular cells that are enlarged in size when compared to normal cells. (D) Primary osteosarcoma tissue treated with SAM showing reversion in cellular pattern. (E) Metastatic osteosarcoma tissue treated with SAM showing no changes in cellular pattern. Scale bar: $100 \mu \mathrm{m}$.

Abbreviation: SAM, S-adenosylmethionine.

is that it has an enlarged proliferative mass of cells with an irregular pattern of tissue arrangement compared to primary osteosarcoma (Figure 1B). Also, some of the cells were displaced to such an extent that the gaps between the clumps of cells were visible (Figure 1C). Upon SAM administration as described in the "Materials and methods" section, there was a remarkable improvement with reduction of proliferative cells, which is evident from the histological observation of primary osteosarcoma (Figure 1D) when compared with nontreated primary osteosarcoma(Figure 1B). Butnopositive improvement was observed in SAM-treated metastatic osteosarcoma (Figure 1E) as was analyzed with nontreated metastatic osteosarcoma (Figure 1C). From that, it was obvious that SAM has a positive effect in the primary stage of osteosarcoma.

\section{Increased expression of Sox2 in primary and metastatic osteosarcoma}

The immunohistochemistry of normal bone tissue with antiSox 2 antibody showed a minimal expression as illustrated in Figure 2A. But in response to osteosarcoma progression, its expression got upregulated (Figure $2 \mathrm{~B}$ and $\mathrm{C}$ ). In the case of primary osteosarcoma, patches of cells showed signals for Sox2 (Figure 2B), whereas in the metastatic form of osteosarcoma, strong signals were visualized from the background of higher proliferative mass of cells (Figure 2C). With SAM administration, osteosarcoma tissue showed histological improvement along with the repressed expression of Sox 2 protein in the primary stage of osteosarcoma (Figure 2D). Following SAM treatment, the osteosarcoma development was controlled in the primary stage with a minimal expression of Sox 2 along with restitution of the cellular arrangement (Figure 2D). But Sox 2 expression was not restricted in the advanced stage of metastatic osteosarcoma (Figure 2E).

\section{Expression studies of TCTP in primary and metastatic osteosarcoma}

To understand the osteosarcoma progression and to crosscheck the results further, the expression pattern of TCTP was studied using immunohistochemistry. The control bone tissue had limited TCTP protein expression (Figure 3A), but upon osteosarcoma development, especially in the primary stages of osteosarcoma, there was an increased TCTP protein expression as shown in Figure 3B. But the advanced metastatic osteosarcoma showed only a restricted expression of TCTP with very mild signals (Figure 3C). After SAM treatment, interestingly, the primary osteosarcoma tissue showed an elevated expression of TCTP, which implies the 

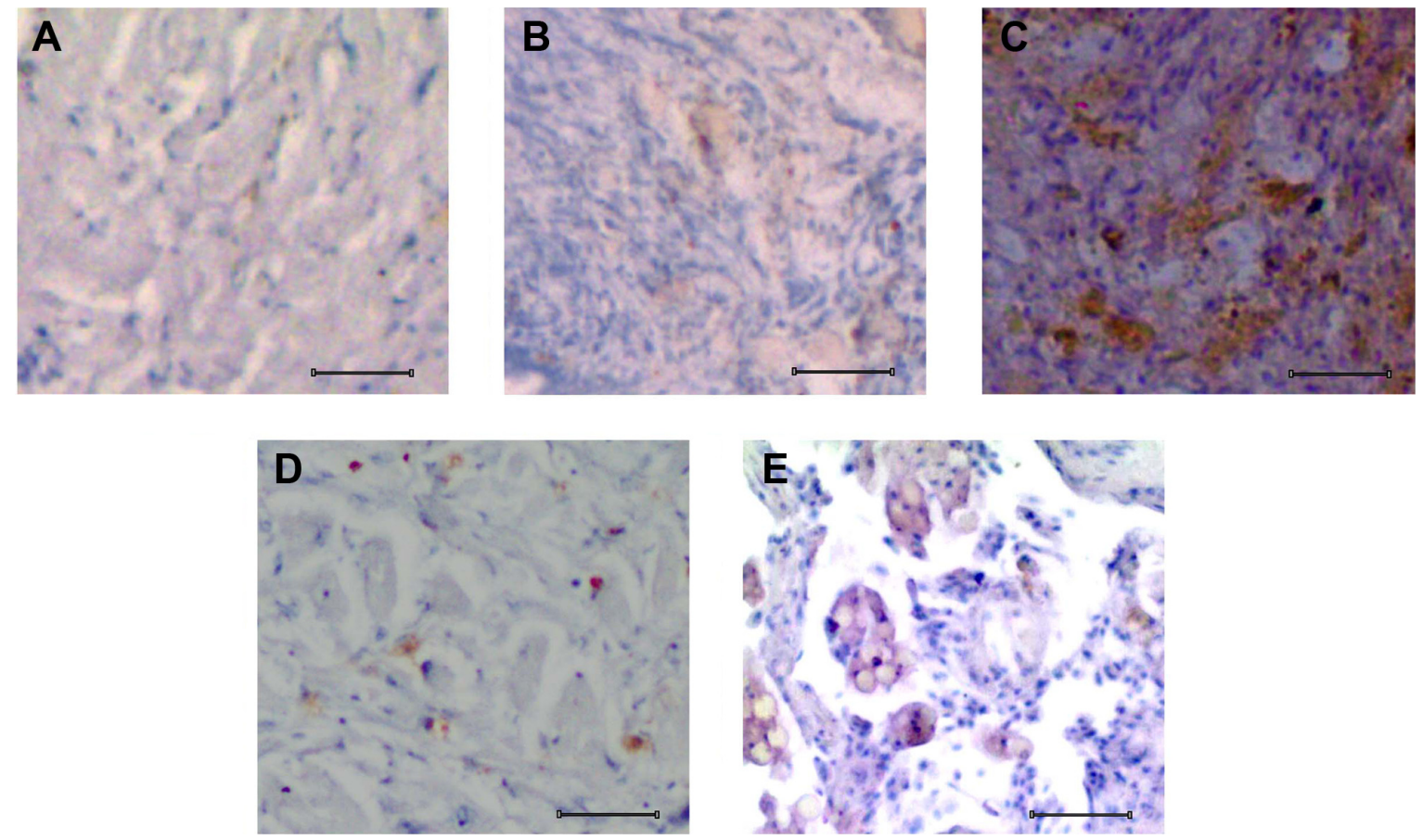

Figure 2 Analysis of Sox2 expression in normal and osteosarcoma tissues.

Notes: (A) Sox2 expression in normal bone tissue of a mouse. (B) Sox2 expression in primary osteosarcoma tissue. (C) Sox2 expression in metastatic osteosarcoma tissue. (D) Sox2 expression in SAM-treated primary osteosarcoma tissue. (E) Sox2 expression in SAM-treated metastatic osteosarcoma tissue. Scale bar: 100 m.

Abbreviation: SAM, S-adenosylmethionine.
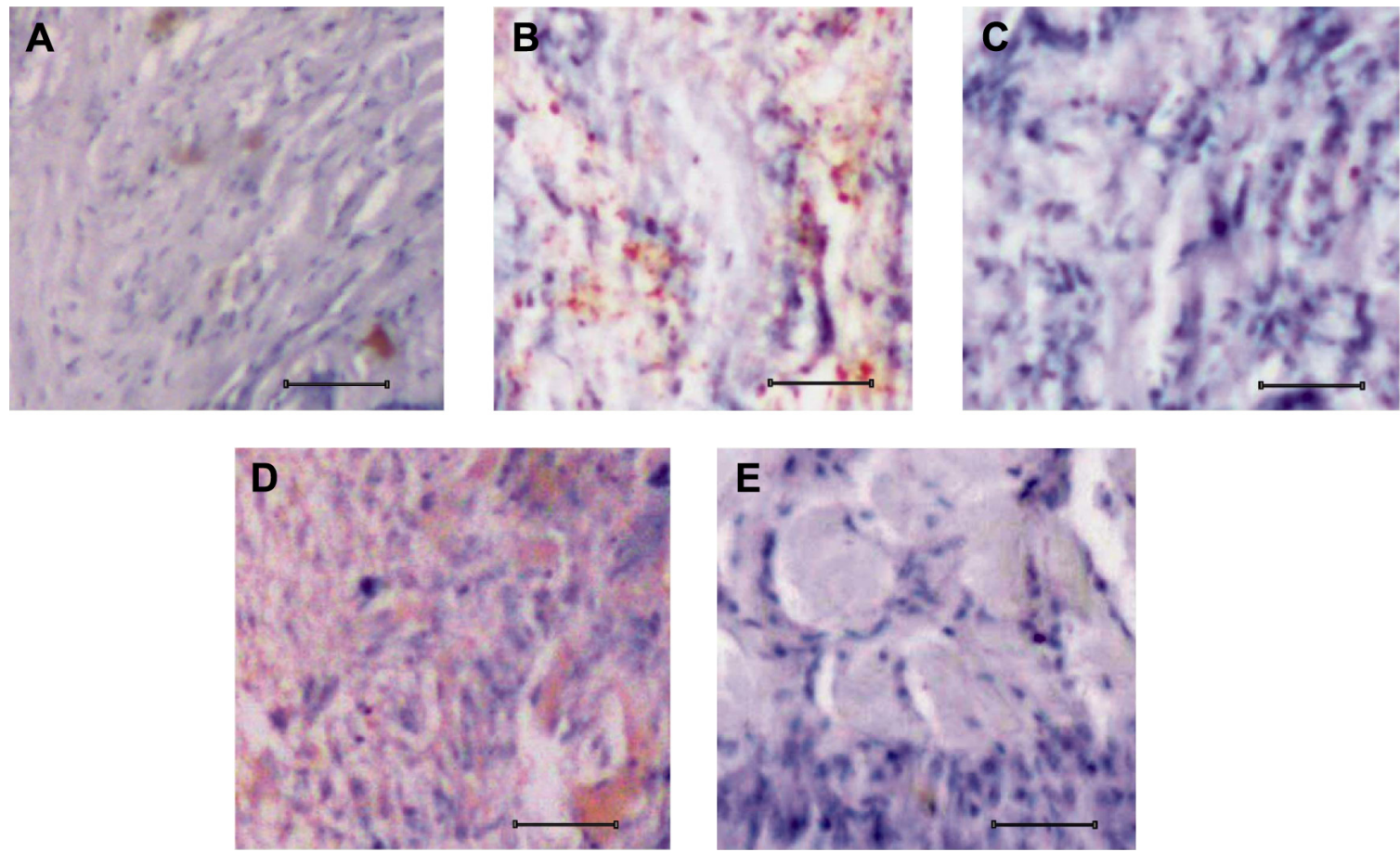

Figure 3 Analysis of TCTP expression in normal and osteosarcoma tissues.

Notes: (A) TCTP expression in normal bone tissue of a mouse. (B) TCTP expression in primary osteosarcoma tissue. (C) TCTP expression in metastatic osteosarcoma tissue. (D) Increased TCTP expression in SAM-treated primary osteosarcoma tissue. (E) TCTP expression in SAM-treated metastatic osteosarcoma tissue. Scale bar: $100 \mu \mathrm{m}$.

Abbreviation: SAM, S-adenosylmethionine. 


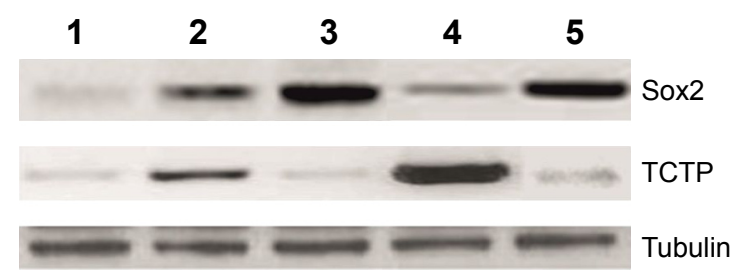

Figure 4 Western blotting analysis of Sox 2 and TCTP expression.

Notes: The protein samples were prepared from SAM-treated osteosarcoma tissue along with various controls and subjected to Western blotting analysis and then stained with anti-Sox 2 antibody and anti-TCTP antibody. Lane I shows Sox 2 and TCTP expression in normal bone tissue samples of mice. Lane 2 represents Sox 2 and TCTP expression in primary osteosarcoma tissue samples. Lane 3 represents Sox 2 and TCTP expression in metastatic osteosarcoma tissue samples. Lane 4 shows Sox2 and TCTP expression in SAM-treated primary osteosarcoma tissue samples. Lane 5 represents Sox 2 and TCTP expression in SAM-treated metastatic osteosarcoma tissue samples. Tubulin was used as a loading control.

Abbreviation: SAM, S-adenosylmethionine.

fight back of cells to prevent osteosarcoma (Figure 3D), but in the case of metastatic osteosarcoma, the TCTP expression showed negative changes that resulted in the development of metastatic form.

\section{Western blotting to analyze Sox2 and TCTP expression}

The results obtained using immunohistochemistry were further validated using Western blotting by comparing the Sox 2 expression with that of TCTP. It is possible to precisely determine the osteosarcoma-regulating proteins in different tissues. The protein samples from normal bone tissue, primary and metastatic osteosarcoma tissues, and SAM-treated tissues were subjected to Western blotting analysis using anti-Sox 2 antibody and anti-TCTP antibody (Figure 3 ). Throughout the experiment, we observed that Sox 2 was correlated with osteosarcoma progression, and upon treatment with SAM, the Sox2 level reduced along with the inhibition of tumor load in primary osteosarcoma, but SAM had no effect on metastatic osteosarcoma. The results with TCTP showed that it had an increased expression in primary osteosarcoma when compared with the normal tissue. But its expression showed some changes in metastatic osteosarcoma; on the other hand, its expression got upregulated in primary osteosarcoma after treatment with SAM (Figure 4).

\section{Discussion}

The osteosarcoma mouse model has the capability to bear a resemblance to human cancer histologically that greatly helps to understand the disease biology. ${ }^{29}$ In the present study, the injected K7M2 cells worked effectively in the well-established $\mathrm{BALB} / c$ strain of mice, ${ }^{30}$ which is widely used in the osteosarcoma mouse model to study the basic science and for clinical investigations. ${ }^{31-34}$ In contrast, it was reported that SAM at a concentration of $150 \mu \mathrm{M}$ through intravenous route resulted in the development of significantly smaller skeletal lesions and at the same time marked reduction in pulmonary metastasis of osteosarcoma as compared to control groups in SCID mice. ${ }^{28}$ But in the present experiment, osteosarcoma-developed $\mathrm{BALB} / \mathrm{c}$ mice had not shown any lesions like SCID mice. It is interesting to note that SAM had different roles in the SCID than in the $\mathrm{BALB} / \mathrm{c}$ mouse strain. The experimental success of developing primary and metastatic osteosarcoma is visualized with an abnormal proliferative mass of cells together with deeply stained clusters of cells (Figure 1B and C). To understand the role of SAM in osteosarcoma, the mice were administered SAM. The role of SAM in inhibiting the primary osteosarcoma (Figure 1D) was effective when compared with the control (Figure 1B). But it had no effective role in inhibiting the later stages of osteosarcoma development (Figure 1E), which implies that the SAM has not much potential to stop metastatic osteosarcoma.

The molecular role of SAM was identified to have an effect on many transcription factors and genes associated with growth and apoptosis. ${ }^{35-37}$ The function of Sox 2 was also well defined in osteosarcoma that its expression is associated with triggering of the proliferative ability of normal bone tissue and the knockout of Sox2 leads to having a control over its proliferation ability. ${ }^{38}$ The present study revealed the role of SAM in controlling the Sox 2 expression. While the osteosarcoma progresses, the Sox 2 also shows overexpression (Figure 2A and C), which implies that the development of osteosarcoma is associated with Sox 2 expression. Similarly, the TCTP downregulation is also associated with osteosarcoma progression (Figures 3 and 4).

The regulation of Sox 2 by SAM is more understandable with the experiment of SAM administration into the osteosarcoma model that inhibits tumor progression along with the downregulation of Sox 2 expression (Figure 2D). SAM methylate-mediated downregulation of TCTP expression does not happen in primary osteosarcoma (Figure 3D), but similar effects occur in metastatic osteosarcoma (Figure 3E). On examining the results of SAM-treated metastatic osteosarcoma along with SAM-treated primary osteosarcoma, the Sox 2 expression is well controlled in the primary form of osteosarcoma because SAM has a control over the initial stage of osteosarcoma but it does not happen in the advanced stage of osteosarcoma. In addition, the results of immunohistochemistry with anti-TCTP antibody illustrate that TCTP has an important role in controlling 
the tumor. In contrast, it was already reported that normal and transformed cells show limited expression of TCTP. ${ }^{39}$ It was also reported that the TCTP levels are downregulated through activation of the tumor suppressor protein $\mathrm{p} 53.22,40$ Immunohistochemistry analysis shows elevated expression of TCTP, and the results coincide with the data illustrating. In addition to human osteosarcoma cells, other cell lines have also expressed the elevated levels of TCTP. ${ }^{41}$

In conclusion, the collective results show that SAM plays a major role in controlling osteosarcoma progression. As a supportive effect of osteosarcoma reversion after SAM administration, we observed downregulation of Sox2 expression along with key upregulation of TCTP protein in primary osteosarcoma. The results obtained in this study are interesting, which promotes the prognosis of osteosarcoma for future investigation.

\section{Acknowledgments}

We thank the institutional review board approval committee and the ethical committee for the successful completion of this project.

\section{Disclosure}

The authors report no conflicts of interest in this work.

\section{References}

1. Picci P. Osteosarcoma (osteogenic sarcoma). Orphanet J Rare Dis. 2007;2:6.

2. Smida J, Baumhoer D, Rosemann M, et al. Genomic alterations and allelic imbalances are strong prognostic predictors in osteosarcoma. Clin Cancer Res. 2010;16(16):4256-4267.

3. Ottaviani G, Jaffe N. The epidemiology of osteosarcoma. In: Jaffe N, Bruland O, Bielack S, editors. Pediatric and Adolescent Osteosarcoma. New York: Springer; 2009:3-13.

4. Tan ML, Choong PF, Dass CR. Osteosarcoma - conventional treatment vs gene therapy. Cancer Biol Ther. 2009;8(2):106-117.

5. Allison DC, Carney SC, Ahlmann ER, et al. A meta-analysis of osteosarcoma outcomes in the modern medical era. Sarcoma. 2012;2012: 704872.

6. Meyers PA. Muramyl tripeptide (mifamurtide) for the treatment of osteosarcoma. Expert Rev Anticancer Ther. 2009;9(8):1035-1049.

7. Szuhai K, Cleton-Jansen A-M, Hogendoorn PC, Bovée JV. Molecular pathology and its diagnostic use in bone tumors. Cancer Genet. 2012;205(5):193-204.

8. Esteller M. Cancer epigenomics: DNA methylomes and histonemodification maps. Nat Rev Genet. 2007;8(4):286-298.

9. Das PM, Ramachandran K, VanWert J, et al. Methylation mediated silencing of TMS1/ASC gene in prostate cancer. Mol Cancer. 2006; 5(1):28.

10. Al-Romaih K, Sadikovic B, Yoshimoto M, Wang Y, Zielenska M, Squire JA. Decitabine-induced demethylation of $5^{\prime} \mathrm{CpG}$ island in GADD45A leads to apoptosis in osteosarcoma cells. Neoplasia. 2008; 10(5):471-480.

11. Cote GM, Choy E. Role of epigenetic modulation for the treatment of sarcoma. Curr Treat Options Oncol. 2013;14(3):454-464.

12. Chiang P, Gordon R, Tal J, et al. S-Adenosylmethionine and methylation. FASEB J. 1996;10(4):471-480.
13. Luka Z, Mudd SH, Wagner C. Glycine N-methyltransferase and regulation of S-adenosylmethionine levels. J Biol Chem. 2009;284(34): 22507-22511.

14. Bale S, Ealick SE. Structural biology of S-adenosylmethionine decarboxylase. Amino Acids. 2010;38(2):451-460.

15. Suzuki MM, Bird A. DNA methylation landscapes: provocative insights from epigenomics. Nat Rev Genet. 2008;9(6):465-476.

16. Böhm H, Benndorf R, Gaestel M, et al. The growth-related protein P23 of the Ehrlich ascites tumor: translational control, cloning and primary structure. Biochem Int. 1989;19(2):277-286.

17. MacDonald SM, Rafnar T, Langdon J, Lichtenstein LM. Molecular identification of an IgE-dependent histamine-releasing factor. Science. 1995;269(5224):688-690.

18. Thaw P, Baxter NJ, Hounslow AM, Price C, Waltho JP, Craven CJ. Structure of TCTP reveals unexpected relationship with guanine nucleotide-free chaperones. Nat Struct Mol Biol. 2001;8(8): 701-704.

19. Yenofsky R, Cereghini S, Krowczynska A, Brawerman G. Regulation of mRNA utilization in mouse erythroleukemia cells induced to differentiate by exposure to dimethyl sulfoxide. Mol Cell Biol. 1983; 3(7):1197-1203.

20. Brioudes F, Thierry A-M, Chambrier P, Mollereau B, Bendahmane M. Translationally controlled tumor protein is a conserved mitotic growth integrator in animals and plants. Proc Natl Acad Sci US A. 2010;107(37): 16384-16389.

21. Gachet Y, Tournier S, Lee M, Lazaris-Karatzas A, Poulton T, Bommer U-A. The growth-related, translationally controlled protein P23 has properties of a tubulin binding protein and associates transiently with microtubules during the cell cycle. J Cell Sci. 1999;112(8): 1257-1271.

22. Tuynder M, Susini L, Prieur S, et al. Biological models and genes of tumor reversion: cellular reprogramming through tpt1/TCTP and SIAH-1. Proc Natl Acad Sc U S A. 2002;99(23):14976-14981.

23. Cans C, Passer BJ, Shalak V, et al. Translationally controlled tumor protein acts as a guanine nucleotide dissociation inhibitor on the translation elongation factor eEF1A. Proc Natl Acad Sci U S A. 2003;100(24): 13892-13897.

24. Chen SH, Wu P-S, Chou C-H, et al. A knockout mouse approach reveals that TCTP functions as an essential factor for cell proliferation and survival in a tissue- or cell type-specific manner. Mol Biol Cell. 2007;18(7): 2525-2532.

25. Zhu W-L, Cheng H-X, Han N, et al. Messenger RNA expression of translationally controlled tumor protein (TCTP) in liver regeneration and cancer. Anticancer Res. 2008;28(3A):1575-1580.

26. Arcuri F, Papa S, Carducci A, et al. Translationally controlled tumor protein (TCTP) in the human prostate and prostate cancer cells: expression, distribution, and calcium binding activity. Prostate. 2004; 60(2):130-140.

27. Khanna C, Khan J, Nguyen P, et al. Metastasis-associated differences in gene expression in a murine model of osteosarcoma. Cancer Res. 2001; 61(9):3750-3759.

28. Parashar S, Cheishvili D, Arakelian A, et al. S-Adenosylmethionine blocks osteosarcoma cells proliferation and invasion in vitro and tumor metastasis in vivo: therapeutic and diagnostic clinical applications. Cancer Med. 2015;4(5):732-744.

29. Ek ET, Dass CR, Choong PF. Commonly used mouse models of osteosarcoma. Crit Rev Oncol Hematol. 2006;60(1):1-8.

30. Schmidt J, Strauß GP, Schön A, et al. Establishment and characterization of osteogenic cell lines from a spontaneous murine osteosarcoma. Differentiation. 1988;39(3):151-160.

31. Wan X, Mendoza A, Khanna C, Helman LJ. Rapamycin inhibits ezrinmediated metastatic behavior in a murine model of osteosarcoma. Cancer Res. 2005;65(6):2406-2411.

32. Terabe M, Khanna C, Bose S, et al. CD1d-restricted natural killer T cells can down-regulate tumor immunosurveillance independent of interleukin-4 receptor-signal transducer and activator of transcription 6 or transforming growth factor- $\beta$. Cancer Res. 2006;66(7):3869-3875. 
33. Barkan D, Kleinman H, Simmons JL, et al. Inhibition of metastatic outgrowth from single dormant tumor cells by targeting the cytoskeleton. Cancer Res. 2008;68(15):6241-6250.

34. Merchant MS, Melchionda F, Sinha M, Khanna C, Helman L, Mackall CL. Immune reconstitution prevents metastatic recurrence of murine osteosarcoma. Cancer Immunol Immunother. 2007;56(7):1037-1046.

35. Lu SC, Mato JM. Role of methionine adenosyltransferase and S-adenosylmethionine in alcohol-associated liver cancer. Alcohol. 2005; 35(3):227-234.

36. Chen H, Xia M, Lin M, et al. Role of methionine adenosyltransferase 2A and S-adenosylmethionine in mitogen-induced growth of human colon cancer cells. Gastroenterology. 2007;133(1):207-218.

37. Li TW, Zhang Q, Oh P, et al. S-Adenosylmethionine and methylthioadenosine inhibit cellular FLICE inhibitory protein expression and induce apoptosis in colon cancer cells. Mol Pharmacol. 2009;76(1): 192-200
38. Basu-Roy U, Ambrosetti D, Favaro R, Nicolis SK, Mansukhani A, Basilico C. The transcription factor Sox2 is required for osteoblast self-renewal. Cell Death Differ. 2010;17(8):1345-1353.

39. Bommer U-A, Thiele B-J. The translationally controlled tumour protein (TCTP). Int J Biochem Cell Biol. 2004;36(3):379-385.

40. Niforou KN, Anagnostopoulos AK, Vougas K, Kittas C, Gorgoulis VG, Tsangaris GT. The proteome profile of the human osteosarcoma U2OS cell line. Cancer Genomics Proteomics. 2008;5(1):63-78.

41. Shen JH, Qu CB, Chu HK, et al. siRNA targeting TCTP suppresses osteosarcoma cell growth and induces apoptosis in vitro and in vivo. Biotechnol Appl Biochem. 2016;63(1):5-14.
OncoTargets and Therapy

\section{Publish your work in this journal}

OncoTargets and Therapy is an international, peer-reviewed, open access journal focusing on the pathological basis of all cancers, potential targets for therapy and treatment protocols employed to improve the management of cancer patients. The journal also focuses on the impact of management programs and new therapeutic agents and protocols on

\section{Dovepress}

patient perspectives such as quality of life, adherence and satisfaction. The manuscript management system is completely online and includes a very quick and fair peer-review system, which is all easy to use. Visit http://www.dovepress.com/testimonials.php to read real quotes from published authors.

Submit your manuscript here: http://www.dovepress.com/oncotargets-and-therapy-journal 